\title{
Patient Preoperative Web Portal for Remote, Self-completed Assessment \& Triage: a Feasibility Study
}

\author{
Matt-Mouley Bouamrane, \\ Akintunde Adeyemo, Marilyn Lennon \\ Department of Computing and Information Sciences \\ University of Strathclyde \\ FirstName.LastName@strath.ac.uk
}

\author{
Stefan Schraag \\ Department of Anaesthesia \\ Golden Jubilee National Hospital \\ stefanschraag@btinternet.com
}

\begin{abstract}
Golden Jubilee National Hospital (GJNH) is a state-of-the art tertiary referral centre, carrying out a range of major surgical procedures on behalf of the National Health Service (NHS) in Scotland. In order to improve the integrated care pathway for patient surgical assessment at GJNH, we conducted a feasibility study for a Preoperative Patient Web portal. We collaborated with the anaesthetic department to design and develop a web based preoperative questionnaire which can be self-completed remotely by patients through a web-browser (patient portal). An initial feasibility study of the system suggests that a simple self-completed preoperative web-based questionnaire could be deployed in practice at GJNH, with potential anticipated benefits including reduced costs and improved patient preoperative triage.
\end{abstract}

\section{Self-Completed Patient Questionnaires, Preoperative Assessment, Feasibility Study}

\section{INTRODUCTION}

Patients undergoing surgery are increasingly older, often have complex chronic morbidities and require careful perioperative management (preoperative assessment, surgical care and post-discharge monitoring). However, a recent report by the National Confidential Enquiry into Patient Outcome and Death in the U.K. found that nearly $20 \%$ of elective high-risk patients were not seen in a preoperative clinic prior to surgery [1].

In collaboration with Golden Jubilee National Hospital (GJNH), we designed and developed a web based preoperative questionnaire which could be self-completed remotely by patients through a web browser (patient portal). We then conducted a feasibility study to assess whether the patient web portal for preoperative assessment could be integrated in the existing patient perioperative pathway at the hospital. We started by conducting detailed requirements gathering through a scoping review of the literature on perioperative information systems, combined with 2 semi structured interview-based field visits to the GJNH perioperative clinic.

Based on the interview data and the literature reviews we developed an electronic version of an existing validated, self-completed patient preoperative questionnaire.
We further enhanced the system by integrating context-based conditional branching to dynamically adapt the content of 'follow-up questions' to the patients' individual responses to previous questions. We also introduced simple error checking mechanisms - such as a questionnaire summary feedback for patients - which is a critical feature for safe clinical use. We further introduced a clinical summary for the preoperative nurses and the ability to perform simple analytics on the patients' medical histories to categorise patients into varying categories of risk (i.e. low vs. high). A heuristic usability study of the questionnaire was conducted with 10 students at the University of Strathclyde. After iterative improvements of the web-based preoperative questionnaire, we collected final usability and feasibility feedback via a structured walkthrough of the system and semistructured interview with 2 members of GJNH (anaesthetist and IT manager). Our feasibility study suggests that a simple self-completed preoperative web-based questionnaire could indeed be deployed in practice at GJNH.

\section{BACKGROUND \& RELATED WORK}

\subsection{Golden Jubilee National Hospital}

Golden Jubilee National Hospital (Clydebank, Scotland) is a state-of-the art tertiary referral centre, carrying out a range of major surgical procedures across the NHS in Scotland. 
In 2013, GJNH provided treatment to over 23,000 patients in 'acute' clinical specialties. As patients are referred to GJNH from across Scotland for treatment, this introduces a number of challenges for patients and clinicians, including:

- the medical records of patients referred to GJNH are not available to the care team despite a personalised surgical risk assessment being mandatory for all patients,

- patients may have to travel to GJNH from afar for a preoperative consultation - and in some cases stay overnight - and the travel expenses for preoperative appointments are currently incurred by the referring NHS health-board,

- following discharge, patients may have to return to GJNH for unnecessary check-ups while others develop complications unknown to the care team because of a lack of a convenient follow-up mechanism.

\subsection{Preoperative Information Systems}

We have previously conducted a scoping review of the literature on Pre-Operative Information Systems (POIS) [2, 3] and report on a representative sample of the systems which have been implemented to date:

\begin{abstract}
- NHS Greater Glasgow and Clyde Preoperative Portal: As part of a study on perioperative information systems in NHS Scotland [4], Bouamrane \& Mair reported on the implementation of a number of clinical preoperative information systems. Among these, the NHS Greater Glasgow and Clyde (GGC) Preoperative Portal was a significant health-board wide regional implementation [5]. The portal has been integrated into the patient perioperative care pathway, allowing hospitals to access a patient medical history via the portal on the health-board intranet. Over 100,000 patient preoperative assessments have now been completed to date via the electronic portal.
\end{abstract}

- NHS Dumfries \& Galloway POIS: As part of the same study, Bouamrane \& Mair identified a standalone preoperative information system developed in NHS Dumfries \& Galloway Royal Infirmary [6]. The study highlighted that the use of a computerised system for managing preoperative documentation substantially transformed clinical practices and facilitated communication and information-sharing among the preoperative multidisciplinary team. The system was developed collaboratively by the hospital preoperative staff and the health-board IT team, resulting in a highly contextualised operationalisation of clinical and information management processes.
- HealthQuiz: is an early electronic-based preoperative patient medical questionnaire [7]. The initial study concluded that an electronic device could collect medical history information of comparable quality to that of a personal interview with a health professional - a finding which has been confirmed by several studies since [8].

\section{- AIMS (University Hospital Giessen - UHG):}

an Anaesthesia Information Management System (AIMS, NarkoData) has been used at UHG for perioperative patient data record keeping since 1995 [9]. The system records all patient anaesthesia related data collected during preoperative assessment and peri-operative and postoperative care in the recovery room.

Patient demographics, data from previous procedures and anaesthesia assessments and laboratory data are imported from the hospital information system. The systems uses rule-based algorithms to suggest the request of additional information when pathologies are identified. Information collected during pre-operative assessment is directly accessible by the anaesthesiologist in the operating theatre.

- HealthQuest (HQ): is a computer-based preoperative questionnaire developed at the Cleveland Clinic [10]. The patient interface is based on touch screen technology and at the time of reporting (2000), 50 workstations were in operation in the clinic. Once the patient has completed the medical questionnaire, the system generates a summary of relevant findings, including personalised anaesthetic and surgical risk assessment as well as corresponding alerts to the multi-disciplinary team. The system also records medications and allergies. Patients are classified into 4 categories of risks, similar to the ASA classification: from healthy (1) to patients with multiple complex medical issues (4). A retrospective analysis of more than 60,000 patients over a 3-year period suggested that the redesigned pre-operative assessment model used at the clinic generated several benefits, including: a reduction of pre-operative patient appointments with an anaesthesiologist, a decrease in the surgical delay rate, a reduction of unnecessary laboratory testing, and a reduction of patient interview time and dissatisfaction with the preoperative assessment process.

- OSCAR (Optimising Surgical Care and Assessment Record): is a pre-operative Decision Support System used since 2005 at the Royal Liverpool and Broadgreen hospitals [11]. The system is an intranet, web-based information system (IS) which records patient medical history as well as some vital signs. 
The system is interfaced with the hospital laboratory system and the hospital patient management system. Tests results are transferred into the IS and the result of the pre-operative assessment is available across the hospital without the need for printing the patient assessment. It generates pre-operative screening test recommendations based on a localised version of the NICE pre-operative guidelines. The systems allows for 2 levels of advice: a "recommendation" if the advice relies on strong evidence and a "suggestion" if the indication is not underpinned by strong evidence.

- Synopsis IQ: This system uses rule-based algorithms to generate a pre-operative summary including the results of key risk scores and recommended patient management strategies. The overall anaesthetic risk computed by the system (cASA) was compared with the ASA (American Society of Anesthesiologists) score estimated (eASA) by anaesthesia caregivers, including anaesthetists, anaesthesia trainees, and physician assistants [12]. The evaluation involved a retrospective analysis of 14349 patient cases and found that the cASA score computed by the system compared favourably with that of estimated by anaesthesia caregivers (eASA), with an overall difference of $1.1 \%$ between the 2 scores.

\section{SYSTEM DESIGN AND DEVELOPMENT}

\subsection{Understanding the Clinical Needs}

In order to define the scope of the project and detailed system specification requirements, 3 meetings where held between February and July 2015 between members of GJNH Department of Anaesthesia and the Department of Computer and Information Sciences (CIS) at the University of Strathclyde. Members of GJNH who attended these meetings included: 2 anaesthetists, a senior preoperative nurse and the IT department project manager. Members of Strathclyde CIS attending the meetings included the project supervisors (MMB \& $M L$ ) and the system developer (AA). Worthy of mention here is that one of the 2 project supervisors (MMB) has over 10 years' experience in designing and implementing electronic information systems in perioperative medicine [1321]. Members of GJNH explained that their main motivation was to optimise the perioperative medicine integrated care pathway by allowing patients scheduled for surgery to become more proactive participants in their care, starting from preoperative assessment. This approach is very much in line with the current Royal College of Anaesthetists Perioperative Medicine programme ${ }^{1}$.

\footnotetext{
${ }^{1}$ https://www.rcoa.ac.uk/perioperativemedicine
}

At GJNH, this entails screening a patient several weeks before surgery in order to identify potential risks associated with surgery. GJNH is the national 'waiting time' hospital, which means that patients are referred to GJNH from across Scotland in order to clear the waiting time back-log at other healthboards. Hence, being able to initially screen some patients remotely via a patient web portal offered many potential benefits, including reducing costs to the patient and the NHS, reducing the need for additional and potentially unnecessary travels, and savings in time and resources. It is important to stress here that the initial triage carried out through the patient web portal would not necessarily mean that patients would no longer attend a preoperative assessment in person at GJNH. However, the idea - after successful trial - would be to triage patients so that only those patients who need to or wish to attend a preoperative assessment in person would have to travel to GJNH.

\subsection{Design Requirements for the Questionnaire}

The core idea behind implementing a Patient Preoperative Web portal is to develop a simple health questionnaire which patients could selfcomplete remotely. The questionnaire should contain enough information for preoperative nurses or anaesthetists to decide whether the patient needed a physical assessment at GJNH prior to surgery or could instead attend the hospital directly for surgery.

A preoperative integrated care pathway used for orthopaedic surgery was provided by the department of anaesthesia to the CIS researchers. However, it was immediately clear that this questionnaire was normally filled by an experienced perioperative nurse during a medical interview with the patient - and therefore was entirely inadequate for remote self-completion by the patients themselves.

During the course of the perioperative information system scoping review, a validated patient selfcompleted preoperative questionnaire was identified [22]. The self-completed preoperative questionnaire had been implemented as part of the Study of Medical Testing for Cataract Surgery (SMTCS), a large randomised clinical trial of 19,250 cataract surgeries that investigated the impact of preoperative medical testing on intraoperative and postoperative medical event rates, albeit as a paper questionnaire and not in digital format as we now intended [22].

The GJNH anaesthetists and CIS researchers agreed to use this validated questionnaire as the basis to test the feasibility of the patient preoperative web portal. 


\subsection{Functional Requirements}

As the results of the requirements elucidation phase, the following functional requirements were established with the web portal stakeholders. The preoperative patient portal should:

- FR1: allow patients to create a user account and modify the account information whenever they want to.

- FR2: allow patients to tailor the assessment (e.g. number of questions they want to appear on each page).

- FR3: implement conditional branching logic within the questionnaire so that assessment questions can be adapted to each patient individual context (i.e. medical history as provided in previous answers).

- FR4: provide patients with an assessment feed-back form for error-checking, which could be generated in pdf format and printed or emailed.

- FR5: provide health professionals with a clinical summary for patient assessment review and generate a pdf document of the clinical summary for archiving.

- FR6: provide health professionals with simple data analytics so aggregated information about patients can be generated (e.g. categories of low risk vs. higher risk patients, types of morbidities, etc.)

- FR7: generate a clinical letter as a pdf document which could be sent to the patient general practitioner for safety and data quality assurance.

\subsection{Development Methodology}

The Patient Preoperative web portal was designed using an Iterative and Incremental software Development Model (IIDM) [23]. IIDM allows independent modules of the system to be developed incrementally over times, so these can be subsequently integrated into a robust and stable system when each module has been satisfactorily completed and tested. Benefits of the IIDM approach include:

- interleaving of the system requirements, specification, design, testing and programming,
- flexibility to incorporate changes into the software functionalities during the development cycle,

- ability to resolve issues identified during each incremental development cycles,

- testing and system refinement are a continuous process integrated into each iteration

- $\quad$ support for the refactoring of codes.

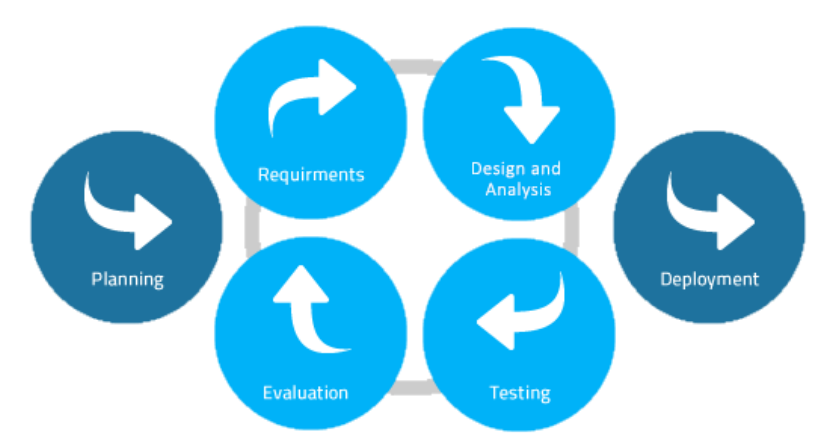

Figure 1: Iterative and Incremental software Development Model, (IIDM)

\subsection{Development Platform}

The Patient Preoperative web portal was developed on the following platform:

- Hypertext Markup Language 5 (HTML5): supports 'intelligent' web forms and was used to define web pages structure

- Cascading Style Sheet 3 (CCS 3): the styling effects can be used to enhance the accessibility of web pages, an important factor for a web portal designed to be used by the general public, which could have a variety of accessibility needs.

- JQuery: is JavaScript library that allows the interaction of client-server scripts with the user, controlling the behaviour of the browser, communicating and modifying web pages content. Furthermore, the syntax permits to manipulate Document Object Model (DOM) elements, the creation of animations and handling events.

- Hypertext Pre-Processor (PHP): was used as the server side scripting language, in order to generate dynamic and interactive web pages.

- MySQL: was used for storing user input.

\subsection{System Architecture}

A standard MVC (Model-View-Controller) architecture was used for the preoperative web portal to allow separation of code, User Interface (UI) in order to guarantee system maintainability and reusability and data integrity. 
MVC also allows convenient and independent update of the core modules of the preoperative portal to be upgraded or replaced separately whenever the systems requirement change.

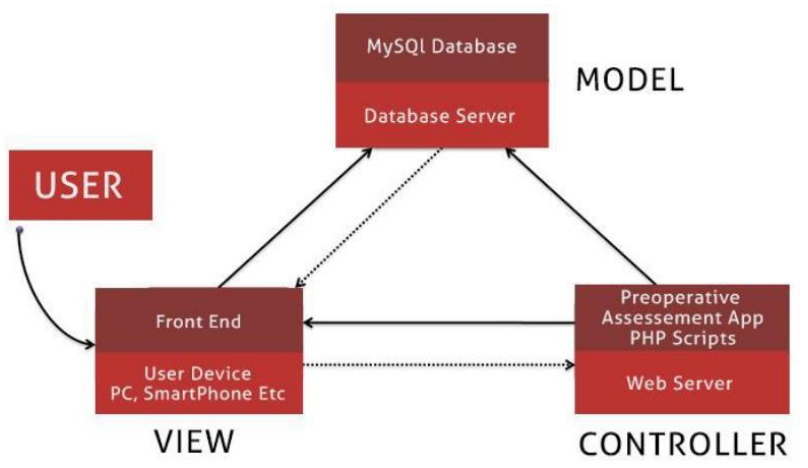

Figure 2: MVC (Model-View-Controller) architecture

The Model represents questionnaire rules and data input and storage. The Model stores and retrieves information, which is transferred to the Controller which it then handed to the View layer.

The Controller intercepts user interaction from the View layer and converts these into actions to be performed by the Model layer.

Finally, the View layer allows data entry and interaction from the user and represents and renders the Model content on the system UI.

As the Preoperative assessment portal is to be used by 2 sets of clinical staff (anaesthetists \& nurses) as well as by patients, the UI design is modularly divided to cater for each category of users. Bootstrap framework is used in designing the UI, because the framework contains sets of cascading style sheets (CSS) which provides style definitions for HTML components. It also includes jQuery plugins, which provides additional functionalities such as dialogs, boxes, tooltips, etc.

\subsection{Overview of system features}

\section{Creating an Adaptive Questionnaire}

The main building blocks of the preoperative assessment system are question and answer classes which are organized in a tree like format. Each 'Question' has an 'Answer' node and each of this answer node can either be an end-node or lead to another 'Question' node (adaptive questionnaire through conditional branching) $[14,15,16]$.

These questions are presented in a sequential manner and two types of property apply to them: a text property which displays the question or answer and the question 'type property' (which define whether the question is adaptive or not).

A conditional branching logic was implemented that allows iterative question instances if a patient answer triggers an adaptive response, in which case a follow-up question may be displayed. To achieve this, a 'getQuestions' method selects and displays additional questions on the UI. JavaScript script is used to trigger the branching of questions and uses the answer provided by the patient.

\section{Have you ever had a heart attack? ?}

$$
\begin{array}{ll}
\text { A. } & \text { Yes } \\
\text { B. No } \\
\text { C. Dont Know }
\end{array}
$$

\section{Next}

\section{Have you ever had a heart attack? ?}

A. Yes In the last 6 months, have you had a heart attack?

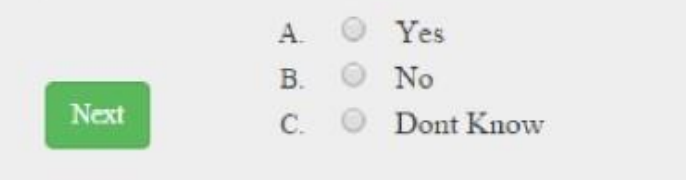

Figure 3: Questionnaire Conditional Branching Logic

\section{Data Analytics}

To give the health professional a bird-eye view of the records of patients, a collection of data analytics was created on a clinician dash board on the portal 'Admin' page. The first form of analytic displays on the panel the number of patients registered for preoperative assessment at the clinic. The next form of analytics displays patients into categories of risk levels. Javascript is used to categorise and display the number of patients and their category of risk into a simple donut shape for quick assessment.

The objective here is to stratify patients based on their risk level by differentiating high risk patients from low risk ones using the answers given by each patient. This provides the health professional with an overview of higher risk patients which may require to be seen in the clinic prior to surgery. Patients with positive answers to critical questions (e.g. history of stroke, heart disease, high blood pressure, complex morbidities, etc.) fall into the category of patients who will most likely need to be seen in person at the clinic prior to surgery. 
Patients who have answered no to critical questions on the other-hand could be scheduled for surgery without the need for an appointment at the preoperative clinic.

\section{YOCRFEDBiCK \\ HetRT CONDTIONS

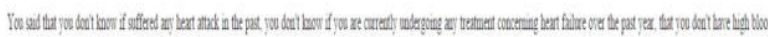

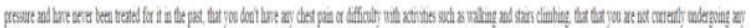

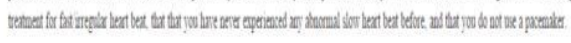 \\ OTHR CONDIIOS

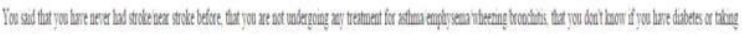

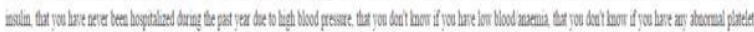

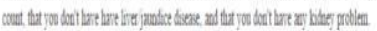 \\ IEDICATIONS

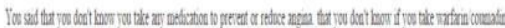

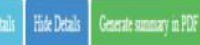

Figure 4: The Patient feedback form can also be generated into a PDF document

\section{Clinical Summaries}

The system requirements included that the preoperative assessment portal should provide short clinical summaries to health professionals and a feed-back summary to the patient who completed the assessment questionnaire.

The purpose of the summaries is to provide essential information using simple natural language processing algorithms on the answers provided. The summaries are illustrated in the portal screenshots below. Furthermore, a clinical letter can also be generated to send to the patient General Practitioner in order to double-check that the information provided by the patient is accurate and consistent with the information held by the GP on the patient primary care electronic medical record.

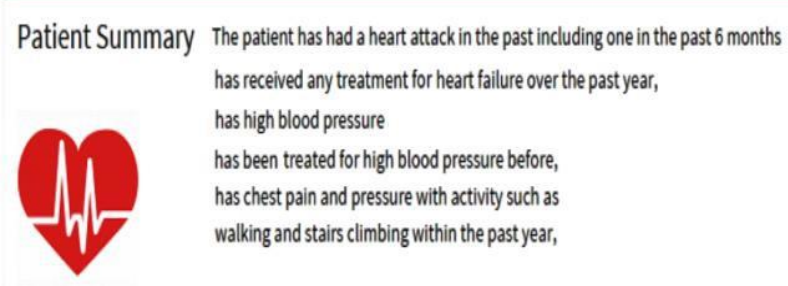

HEART CONDITIONS

Figure 5: Excerpt of the Clinical Summary generated for the Health professionals

\section{EVALUATION}

To test the preoperative assessment system, two types of software and system tests were carried out: source code testing and load performance test. For the source code, a manual test was used to test the main methods. While BlazeMeter was used to test the load performance of the system.

A system usability evaluation was also conducted with 10 students of the University of Strathclyde and a heuristic evaluation with 2 healthcare professionals at GJNH. We will now present the usability evaluation in more detail.

\subsection{Experimental Set-Up}

Fourth year students at the University of Strathclyde were invited to participate in the web portal usability evaluation and 10 were selected to participate in the usability study. Ten was considered an appropriate number of participants to identify the core usability issues based on the findings by Nielsen and Landauer [24] which recommend that using 2 sets of 5 users in subsequent evaluation batches will usually allow to identify the majority of usability issues in web design. Participants were asked to complete the assessment questionnaire as if they were themselves scheduled to attend an appointment at a hospital. The experiment had no time limit and participants could spend as much time as they wished on the questionnaire. Once they completed the task, they were asked to fill in a System Usability Scale (SUS) questionnaire [25].

The SUS questionnaire has 10 questions with a 5 point Likert scale with items $1,3,5,7$ and 9 positively worded and items 2, 4, 6, 8 and 10 negatively worded. This is done to minimize acquiescence and biased responses. Acquiescence bias occurs when a survey question is passively agreed to by a particular participant while the extreme bias response occurs when the available answers selected by the respondent is the most extreme [26]. A research performed by Tullis and Stetson shows that the SUS provides a superior assessment when it comes to website usability and learnability, when compared to other questionnaires [27].

An overall SUS score provided a measure of the composite usability of the system. To calculate the overall SUS score, the contribution score of each item is summed. The score contribution of items 1 , $4,5,7$ and 9 is the scale position of each item minus 1 , while the score contribution of items 2, 4, 6,8 and 10 is 5 minus the scale position of each item. The sum of scales is then multiplied by 2.5 to get the overall value of the System Usability (SU). 
Our usability study provided the average SUS score of $71.4 \%$. The aggregate results of the SUS survey are provided in Figure 6 at the end of this article.

\subsection{Participants' Feedback}

In addition to the SUS survey, a researcher (AA) collected informal verbal feedback from the study participants at the end of the experiment. These comments were then examined for emerging themes (positive and negative) in order to organise and interpret the qualitative comments.

The participants found the evaluation tasks straightforward as they were able to perform the assessment with just a few clicks (i.e. 'YES / NO / DON'T KNOW' bullet lists options). They were able to navigate through the system with ease as information was presented in a simple and logical order and the questionnaire was short and could be completed in a relatively short amount of time.

Overall, participants found the experiment interesting as they found the health assessment portal an application that they could see themselves potentially use in future as part of their health-care journeys. Others found the Natural Language Processing (NLP) feed-back displayed at the end of the questionnaire particularly useful.

Some improvements were suggested however, including the following recommendations:

- Navigation feedback: some participants suggested adding a questionnaire progress bar. Although the questionnaire is short, the participants themselves were not necessarily aware of this when first completing the assessment,

- Feedback Option: some participants suggested to add the option of having a full feedback form in addition to the feedback summary that was initially provided (for archiving purposes).

These recommendations were subsequently integrated into the final iteration of the portal.

\subsection{Health Professionals' Feedback}

In addition to the SUS survey described above, we also conducted a heuristic evaluation [28] of the web portal with two key members of the GJNH department of anaesthetic who had participated in the initial portal design requirements specification.

This method categorises potential usability issues into 10 categories: I. Visibility of the system status; II. Match between system and real world; III. User control and freedom; IV. Consistency and standards and finally: V. Error prevention; VI. Recognition rather than recall; VII. Flexibility and efficiency of use; VIII. Aesthetic and minimalist design; IX. Help users recognize and recover from errors and finally: X. Help and documentation.

The health professional evaluation was carried out to assess the system along these 10 heuristic dimensions. Two members of GJNH took part in this assessment (one anaesthetist and the IT project manager). They were provided with a web link to the portal and associated documentation. Feed-back was then collected verbally and by email. Overall, the two participants felt that the preoperative assessment system fulfilled the specifications which had been initially agreed between GJNH and the University of Strathclyde. The feedback was again categorised thematically to understand what the main positive and negative comments were along the ten usability dimensions detailed above.

The anaesthetist provided the following feed-back:

- Anaesthetists 1: 'It's a very nice work you did and I like it. It is fairly intuitive and clearly presented...'

However, the anaesthetist also had some reservations about the terminology used in the questionnaire, with the concern that it might be too clinical at times and that symptoms of the disease or condition should be asked instead:

- Anaesthetists 1: 'There are a few questions that the patient might not know the meaning, like heart failure. When a technical term is used, it might be easier to check on the typical symptoms rather if he thinks he has the disease or condition. So, for example regarding heart failure, it would be shortness of breath, or swollen ankles or previous treatment with water tablets...'

The anaesthetist also suggested expanding the scope of the questionnaire, as he considered the questions to be overly focused on cardio-vascular diseases.

$\mathrm{He}$ suggested that more generic questions on previous anaesthetic problems experienced by the patients and questions on allergies would need to be added to the preoperative assessment questions in order to allow for a more complete patient assessment.

- Anaesthetists 1: 'As far as I can see, your questions were very much focused to identify a cardio-vascular risk in the patients. To create a full pre-operative profile, questions on previous anaesthetic problems or allergies would need to be added...' 
As for the 2 previous suggestions, we must highlight that - as we have previously mentioned the exact wording of the questions used in the web questionnaire were directly extracted from a validated patient self-completed questionnaire [22].

Consequently, if the wording of some questions had to be changed or additional questions were to be introduced in the patient web portal, then a novel patient questionnaire validation study would need to be completed at GJNH. In order to provide additional support for the questionnaire completion, we did add however a help menu besides the questions so that medical terms could be explained using simple terms, without changing the specific wording of a question.

\section{DISCUSSION \& FUTURE WORK}

Our feasibility study carried out in collaboration between GJNH suggests that a preoperative web portal could be integrated in the patient perioperative pathway at the hospital. While the literature review we conducted identified a number of existing clinical perioperative information systems (section 2.2), none of these currently actively include patients proactively assisting in the assessment by self-completing a preoperative questionnaire remotely via a web portal.

We conducted a usability study with students and heuristic evaluation with GJNH health professionals, suggesting that our prototype system could now been deployed as a part of a pilot study. While our results to date are so far promising, many challenges still lie ahead: these include which hospital IT system to integrate the patient portal with (hospital patient administration system, electronic medical record, hospital portal or a dedicated perioperative information system), safety and security of the application, and accessibility and acceptability for the hospital patients.

\section{REFERENCES}

[1] Findlay G Goodwin A, Protopapa K, Smith N, Mason $M$ : Knowing the risk: A review of the peri-operative care of surgical patients, A report by the National Confidential Enquiry into patient outcome and death (2011). available at http: //www.ncepod.org.uk/2011report2/ downloads/POC_fullreport.pdf

[2] M.-M. Bouamrane, C. Macdonald, I. Ounis, and F. Mair. Protocol-driven Searches for Medical \& Health- Sciences Systematic Reviews. In Proceedings of the 3rd Conference on Theory of Information Retrieval, ICTIR' 11, Bertinoro, Italy, Springer LNCS, September 2011.
[3] Bouamrane MM, Mair F: Managing complexity in pre-operative information management systems. In Proceedings of Managing Interoperability and Complexity in Health Systems, MIXHS'11, 20th ACM Conference on Information and Knowledge Management, CIKM 2011 Glasgow, United Kingdom, 2011. New York, NY, USA: ACM; 2011:3-10.

[4] Bouamrane MM, Mair F: A study of Information Management in the Patient Surgical Pathway in NHS Scotland. In Studies in Health Technology and Informatics. MEDINFO 2013. Proceedings of the 14th World Congress on Medical and Health Informatics, Medinfo 2013, Volume 192. Copenhagen, Denmark: IOS Press; 2013:557561.

[5] Bouamrane MM, Mair F: Implementation of an integrated preoperative care pathway and regional electronic clinical portal for preoperative assessment. BMC Medical Informatics and Decision Making 2014, 14:93 doi:10.1186/1472-6947-14-93

[6] Bouamrane MM, Mair F: A study of clinical and information management processes in the surgical pre-assessment clinic. BMC Med Inform Decis Making 2014, 14(22)

[7] M. Roizen, D. Coalson, R. Hayward, J. Schmittner, R. Thisted, J. Apfelbaum, C. Stocking, C. Cassel, P. Pompei, D. Ford, and E. Steinberg. Can Patients Use an Automated Questionnaire to Define Their Current Health Status? Medical Care, 30(5):MS74-MS84, 1992.

[8] J. Bachman. The patient-computer interview: a neglected tool that can aid the clinician. Mayo Clinic Proceedings, 78:67-78, 2003.

[9] C. Fuchs, L. Quinzio, M. Benson, A. Michel, R. R"ohrig, B. Quinzio, and G. Hempelmann. Integration of a handheld based anaesthesia rounding system into an anaesthesia information management system. International Journal of Medical Informatics, 75(7):553-563, 2006.

[10] B. Parker, J. Tetzlaff, D. Litaker, and W. Maurer. Redefining the preoperative evaluation process and the role of the anesthesiologist. Journal of Clinical Anesthesia, 12(5):350-356, 2000.

[11] B. Murthy, S. Lake, and A. Fisher. Evaluation of a decision support system to predict preoperative investigations. British Journal of Anaesthesia, 100(3):315-321, 2008.

[12] X. Zuidema, R. Tromp Meesters, I. Siccama, and P. Houweling. Computerized model for preoperative risk assessment. British Journal of Anaesthesia, 2011. 
[13] M.-M. Bouamrane, M. McGee-Lennon, S. Brewster, and F. Mair. Using Process-Mapping to Design Integrated Health Information Management Systems. In Proceedings of the 24th IEEE International Symposium on Computer-Based Medical Systems, CBMS'11, Intelligent Patient Management Special Track, Bristol, U.K., June, IEEE Computer Society Press, 2011.

[14] M.-M. Bouamrane, A. Rector, and M. Hurrell. Gathering precise patient medical history with an ontologydriven adaptive questionnaire. In Proceedings of the 21st IEEE International Symposium on Computer- Based Medical Systems, CBMS '08. , 539-541, June 2008.

[15] M.-M. Bouamrane, A. Rector, and M. Hurrell. Ontology-driven adaptive medical information collection system. In Foundations of Intelligent Systems, volume 4994 of Lecture Notes in Computer Science, 574- 584. Springer Berlin / Heidelberg, 2008.

[16] M.-M. Bouamrane, A. Rector, and M. Hurrell. Using ontologies for an intelligent patient modelling, adaptation and management system. In Proceedings of Ontologies \& Databases, ODBASE'08, OTM 2008, volume 5332 of Lecture Notes in Computer Science, 1458-1470. Springer Berlin / Heidelberg, 2008.

[17] M.-M. Bouamrane, A. Rector, and M. Hurrell. Development of an ontology for a preoperative risk assessment clinical decision support system. In $P$ roceedings of 22nd IEEE International Symposium on Computer- Based Medical Systems, CBMS'2009, 1-6, 2009.

[18] M.-M. Bouamrane, A. Rector, and M. Hurrell. A hybrid architecture for a preoperative decision support system using a rule engine and a reasoner on a clinical ontology. In Proceedings of the 3rd International Conference on Web Reasoning and Rule Systems, RR'09, 242-253, Berlin, Heidelberg, 2009. Springer-Verlag.

[19] M.-M. Bouamrane, A. Rector, and M. Hurrell. Semi-automatic generation of a patient preoperative knowledge-base from a legacy clinical database. In Proceedings of Ontologies \& Databases, ODBASE 2009, OTM'09, 12241237, Berlin, Heidelberg, 2009. SpringerVerlag.

[20] M.-M. Bouamrane, A. Rector, and M. Hurrell. Experience of using OWL ontologies for automated inference of routine pre-operative screening tests. In Proceedings of the 9th international semantic web conference on The semantic web - Volume Part II, ISWC'10, 5065, Berlin, Heidelberg, 2010. Springer-Verlag.

[21] M.-M. Bouamrane, A. Rector, and M. Hurrell. Using OWL ontologies for adaptive patient information modelling and preoperative clinical decision support. Knowledge and Information Systems, 1-14, 2010.

[22] Sherman W Reeves, James M Tielsch, Joanne Katz, Eric B Bass, Oliver D Schein, A selfadministered health questionnaire for the preoperative risk stratification of patients undergoing cataract surgery, American Journal of Ophthalmology, Volume 135, Issue 5, May 2003, Pages 599-606

[23] Craig Larman, Victor R. Basili, Iterative and Incremental Development: A Brief History, Computer, vol. 36, no. 6, pp. 47-56, June, 2003, IEEE Computer Society.

[24] Jakob Nielsen and Thomas K. Landauer. 1993. A mathematical model of the finding of usability problems. In Proceedings of the INTERACT '93 and $\mathrm{CHI}$ '93 Conference on Human Factors in Computing Systems (CHI '93). ACM, New York, NY, USA, 206-213.

[25] Brooke, J.: SUS: A "Quick and Dirty" Usability Scale. In: Jordan, P.W., Thomas, B., Weerdmeester, B.A., McClelland (eds.) Usability Evaluation in Industry, pp. 189-194. Taylor \& Francis, London (1996)

[26] Van Vaerenbergh, Y. and Thomas, T.D. (2013) 'Response styles in survey research: A literature review of antecedents, consequences, and remedies'. International Journal of Public Opinion Research, 25 (2), pp.195-217

[27] TS Tullis, JN Stetson. A comparison of questionnaires for assessing website usability. Usability Professional Association Conference, 2004 - comcast.net

[28] Nielsen, J. Heuristic evaluation. In Nielsen, J., and Mack, R. L. (Eds.), Usability Inspection Methods, John Wiley \& Sons, New York, NY, 1994, 25-64. 


\begin{tabular}{|c|c|c|c|c|c|}
\hline $\begin{array}{c}\text { SUS } \\
\text { Questions }\end{array}$ & $\begin{array}{l}\text { STRONGLY } \\
\text { DISAGREE }\end{array}$ & DISAGREE & $\begin{array}{l}\text { DISAGREE } \\
\text { NOR } \\
\text { AGREE }\end{array}$ & AGREE & $\begin{array}{l}\text { STRONGLY } \\
\text { AGREE }\end{array}$ \\
\hline $\begin{array}{l}\text { I think that I would like to use the } \\
\text { system frequently }\end{array}$ & 0 & 1 & 1 & 6 & 2 \\
\hline $\begin{array}{l}\text { I found the system unnecessarily } \\
\text { complex }\end{array}$ & 3 & 4 & 2 & 1 & 0 \\
\hline $\begin{array}{l}\text { I thought the system was easy to } \\
\text { use }\end{array}$ & 0 & 0 & 1 & 4 & 5 \\
\hline $\begin{array}{l}\text { I think that I would need the } \\
\text { support of a technical person to be } \\
\text { able to use the system }\end{array}$ & 4 & 5 & 0 & 0 & 1 \\
\hline $\begin{array}{l}\text { I found the various functions in } \\
\text { the system were well integrated }\end{array}$ & 0 & 1 & 3 & 4 & 3 \\
\hline $\begin{array}{l}\text { I thought there was too much } \\
\text { inconsistency in the system }\end{array}$ & 5 & 3 & 1 & 1 & 0 \\
\hline $\begin{array}{l}\text { I would imagine that most people } \\
\text { would learn to use the system } \\
\text { very quickly }\end{array}$ & 0 & 0 & 0 & 7 & 3 \\
\hline $\begin{array}{l}\text { I found the system not very } \\
\text { intuitive }\end{array}$ & 1 & 4 & 3 & 2 & 0 \\
\hline $\begin{array}{l}\text { I think the system response time is } \\
\text { satisfactory }\end{array}$ & 0 & 1 & 1 & 3 & 5 \\
\hline $\begin{array}{l}\text { I needed to learn a lot of things } \\
\text { before I could get going with the } \\
\text { system }\end{array}$ & 3 & 5 & 1 & 1 & 0 \\
\hline
\end{tabular}

Figure 6: Results of the Preoperative Portal SUS usability survey with $n=10$ participants 